\title{
Cognitive Pretesting and pretest of the STRAIN questionnaire to elaborate work-related stress of health care staff in Switzerland Verständlichkeitsprüfung und Pretest des STRAIN- Fragebogens zur Erhebung der Arbeitsbelastung bei Gesundheitsfachpersonen in der Schweiz
}

\author{
Christoph Golz ${ }^{*}$, Karin Anne Peter ${ }^{1}$, \\ Sabine Hahn' \\ ${ }^{1}$ Berner Fachhochschule, Departement Gesundheit, \\ 3008 Bern, Switzerland \\ *christoph.golz@bfh.ch
}

Received 30 April 2018, accepted 11 July 2018

\begin{abstract}
The workforce shortage in health care is a major challenge worldwide. In Switzerland, this shortage is not only demographically caused but also due to workload. Adequate measures require a good data basis. The aim of this study is to test instrument specially developed for the health professions to measure work-related stress in practice. Cognitive pretesting was used for the evaluation. Overall, 18 semi-structured single-interviews with nurses and nursing assistants were conducted. In two rounds, the questionanswer-process was analysed for 11 questions. The STRAIN questionnaire was then pretested by 17 health professionals. In the first round, 42 problems were identified based on the phases of the "Cognitive Aspects of Survey Methodology". Of these, $57 \%$ were comprehension problems. This was shown by the polysemantic usage or unknowingness of terms. Further $38 \%$ were problems with the answer categories, such as a missing category or an inappropriate formulation. Further questions were modified through a final pretest. The uniform comprehensibility has been achieved. Due to the questionnaire length, an online tool with a save function should be used. The results of this study are similar to those of comparable studies, which also show the most common problems with understanding. This is due to changes of concepts' meanings over time and culture. A cognitive pretest is therefore recommended, especially for a heterogeneous target group such as health professions.
\end{abstract}

\begin{abstract}
Der Fachkräftemangel im Gesundheitswesen ist eine grosse Herausforderung weltweit. In der Schweiz ist neben den demografisch bedingten Ursachen auch die Arbeitsbelastung ein gewichtiger Grund für diesen Mangel. Adäquate Gegenmassnahmen zur gezielten Verbesserung brauchen eine gute Datengrundlage. Ziel dieser Studie ist es, ein für die Gesundheitsberufe entwickeltes Messinstrument zur Erfassung arbeitsbedingter Belastung in der Praxis zu testen. Die Verständlichkeit des Fragebogens wurde mittels kognitiven Interviews bei 18 Pflege- und Betreuungspersonen in zwei Durchgängen evaluiert und anschliessend mittels Pretest bei 17 Gesundheitsfachpersonen getestet. Im ersten Durchgang wurden 42 Probleme anhand der Phasen der "Cognitive Aspects of Survey Methodology“ identifiziert. $57 \%$ waren Verständnisprobleme, die sich durch Mehrdeutigkeit oder Unbekanntheit einzelner Begriffe zeigten. Weitere 38 \% stellten Probleme mit den Antwortkategorien dar, wie das Fehlen einer Kategorie oder einer unpassenden Formulierung. Beim abschliessenden Pretest konnten weitere Fragen modifiziert werden. Insgesamt konnte jedoch nach dem Pretest eine einheitliche Verständlichkeit erreicht werden. Aufgrund der Fragebogenlänge sollte ein Online-Tool mit der Funktion zum Zwischenspeichern verwendet werden. Die Ergebnisse decken sich mit denen vergleichbarer Validierungen, die ebenfalls am häufigsten Probleme beim Verstehen aufzeigen. Dies scheint bedingt durch Kultur und Zeit, denn Begriffe können ihre Bedeutung verändern.
\end{abstract}

\section{Keywords}

cognitive pretesting - work-related stress - STRAIN study - health professions

\section{Keywords}

kognitives Pretesting - Arbeitsbelastung - STRAIN-Studie - Gesundheitsberufe

\section{EINLEITUNG}

Der Fachkräftemangel im Gesundheitswesen ist ein globales Problem. Auch in der Schweiz werden bis zum Jahr 2030 in der Pflege, der personalstärksten Berufsgruppe im Gesundheitswesen, ca. 109‘000 Fachund Hilfspersonen fehlen (Merçay, Burla \& Widmer,
2016). Hauptsächlich werden der Langzeitbereich sowie die ambulanten Dienste vom Mangel betroffen sein (Merçay et al., 2016). Auch in den medizinisch therapeutischen Berufen (Rüesch et al., 2014) sowie dem ärztlichen Dienst (Bolliger, Golder, Jans \& Rüefli, 2016) wird ein Mangel für die nächsten Dekaden prognostiziert. Dieser Fachkräftemangel führt dazu, dass 
die anfallende Arbeit von weniger Personal bewältigt werden muss, was zu einer physischen, psychischen und sozialen Mehrbelastung führen kann (Aiken et al., 2012). Die Belastung am Arbeitsplatz kann sich zudem durch soziodemografische Faktoren (Bsp.: alleinerziehend, Angehörigenpflege, Migrationshintergrund) und politische Rahmenbedingungen (Bsp.: fehlende Kindergrippen, teures Gesundheitssystem) verstärken (Hahn, Richter, Beck \& Thilo, 2013). Auch Faktoren aus dem beruflichen Umfeld, wie die betriebliche Organisation (Bsp.: Schichtarbeit, fehlende Personalförderung) und das soziale Klima bei der Arbeit (Bsp.: Mobbing, fehlende Fehlerkultur) können die Belastung am Arbeitsplatz fördern (Glazer \& Beehr, 2005; Hahn et al., 2013; Suadicani, Olesen, Bonde \& Gyntelberg, 2014). Weiter, kann die Belastung am Arbeitsplatz einen direkten und indirekten Einfluss auf die persönliche Gesundheit (Martikainen, Bartley \& Lahelma, 2002) sowie negative Auswirkungen auf die Arbeitszufriedenheit haben (Aiken et al., 2012). Aiken et al. (2012) stellten fest, dass $21 \%$ der Pflegefachpersonen in der Schweiz unzufrieden mit ihrer Arbeit sind. Gemäss Wenderlein und Schochat (2003) können sich zwei Drittel der Pflegekräfte nicht vorstellen, bis zum Rentenalter im Beruf zu bleiben. $15 \%$ der Pflegefachpersonen wiesen bereits 2012 erhöhte Burnout-Werte auf (Aiken et al., 2012). Ferner leidet insgesamt ein Drittel der Pflegefachpersonen an akuten bzw. chronischen Rückenschmerzen (Schubert et al., 2005). Die Folgen sind krankheitsbedingte Absenzen sowie hohe Fluktuationsraten (Michie \& Williams, 2003; Suadicani et al., 2014). Internationale Studien zeigen auf, dass Faktoren, wie Mobbing, fehlende Sinnhaftigkeit der Arbeit, fehlende Entscheidungsfreiheit, fehlende Unterstützung etc. sich auf die Gesundheit der Mitarbeitenden belastend auswirken können (Hasselhorn, Müller, Tackenberg, Kümmerling \& Simon, 2005; Suadicani et al., 2014).

In der Schweiz sowie international liegen zum Thema Arbeitsbelastung vorwiegend Daten über Pflegefachpersonen vor. Zu den Belastungsfaktoren von Gesundheitsfachpersonen und in der interprofessionellen Zusammenarbeit sind die Erkenntnisse unzureichend (Hahn et al., 2013). Um gezielte und effiziente Interventionen zur Reduktion von Belastungen am Arbeitsplatz im Schweizer Gesundheitswesen identifizieren und die Datenlage verbessern zu können, fehlen auf das Schweizer Gesundheitswesen angepasste Erhebungsinstrumente.

\section{ZIEL}

Das nationale Forschungsprojekt „Work-related Stress among health professionals in Switzerland" (STRAIN) ist eine Interventionsstudie zur Reduktion der Arbeitsbelastung von Gesundheitsfachpersonal in der Schweiz (Berner Fachhochschule, 2017). Die Intervention richtet sich an das mittlere und obere Management, da diese Personen zuständig sind für angemessene Rahmenbedingungen am Arbeitsplatz und die Unterstützung sowie das Coaching der Mitarbeitenden. Im Rahmen dieser Studie wurde ein Fragebogen, basierend auf validen und reliablen Skalen, zur Erfassung der Arbeitsbelastung entwickelt. Zudem wurde der STRAIN-Fragebogen mit spezifischen Fragen (bspw. bezüglich Rahmenbedingungen im Schweizer Gesundheitswesen) ergänzt. Ziel dieser Studie ist es nun, die neu entwickelten und angepassten Fragen und Antwortkategorien des STRAIN-Fragebogens auf einheitliche Verständlichkeit und Augenscheinvalidität bei Schweizer Gesundheitsfachpersonen zu testen.

\section{METHODE}

Damit eine valide Erhebung erfolgen kann, müssen Fragebögen auf Passung zu Setting und Zielgruppe auf Verständlichkeit evaluiert werden. Die Augenscheinvalidität und die einheitliche Verständlichkeit wurden mittels kognitiven Interviews und eines Pretests überprüft und einzelne Fragen an das Sample adaptiert. In Abbildung 1 ist das Vorgehen schematisch dargestellt.

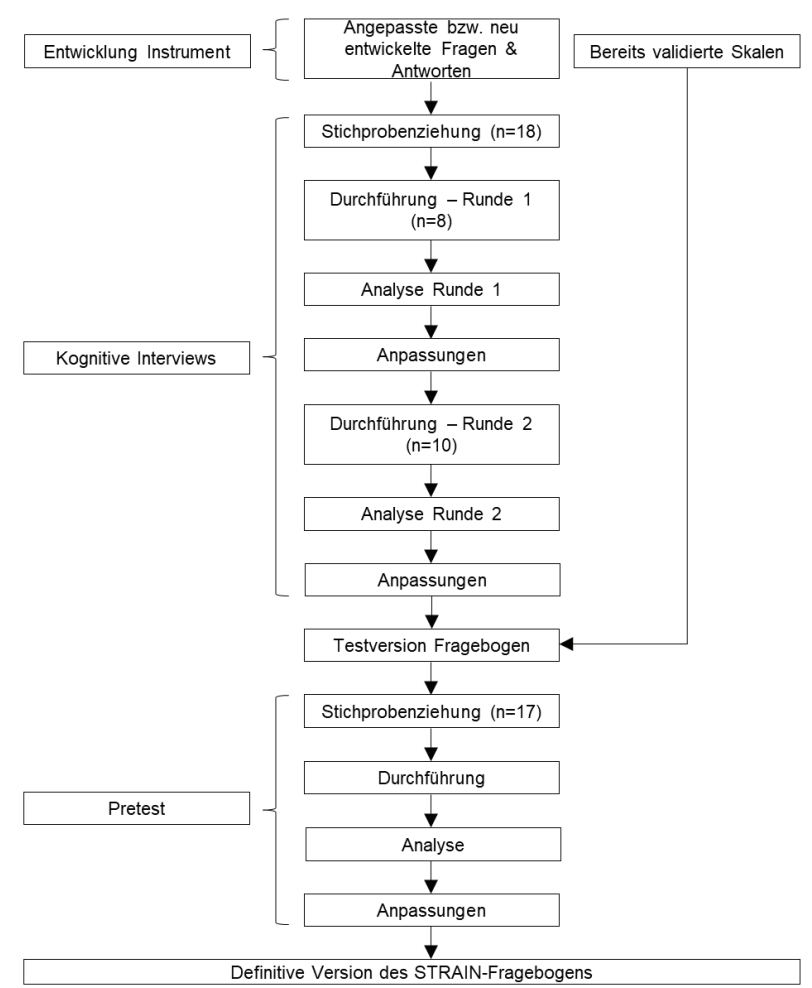

Abbildung 1: Vorgehen Entwicklung STRAIN-Fragebogen 


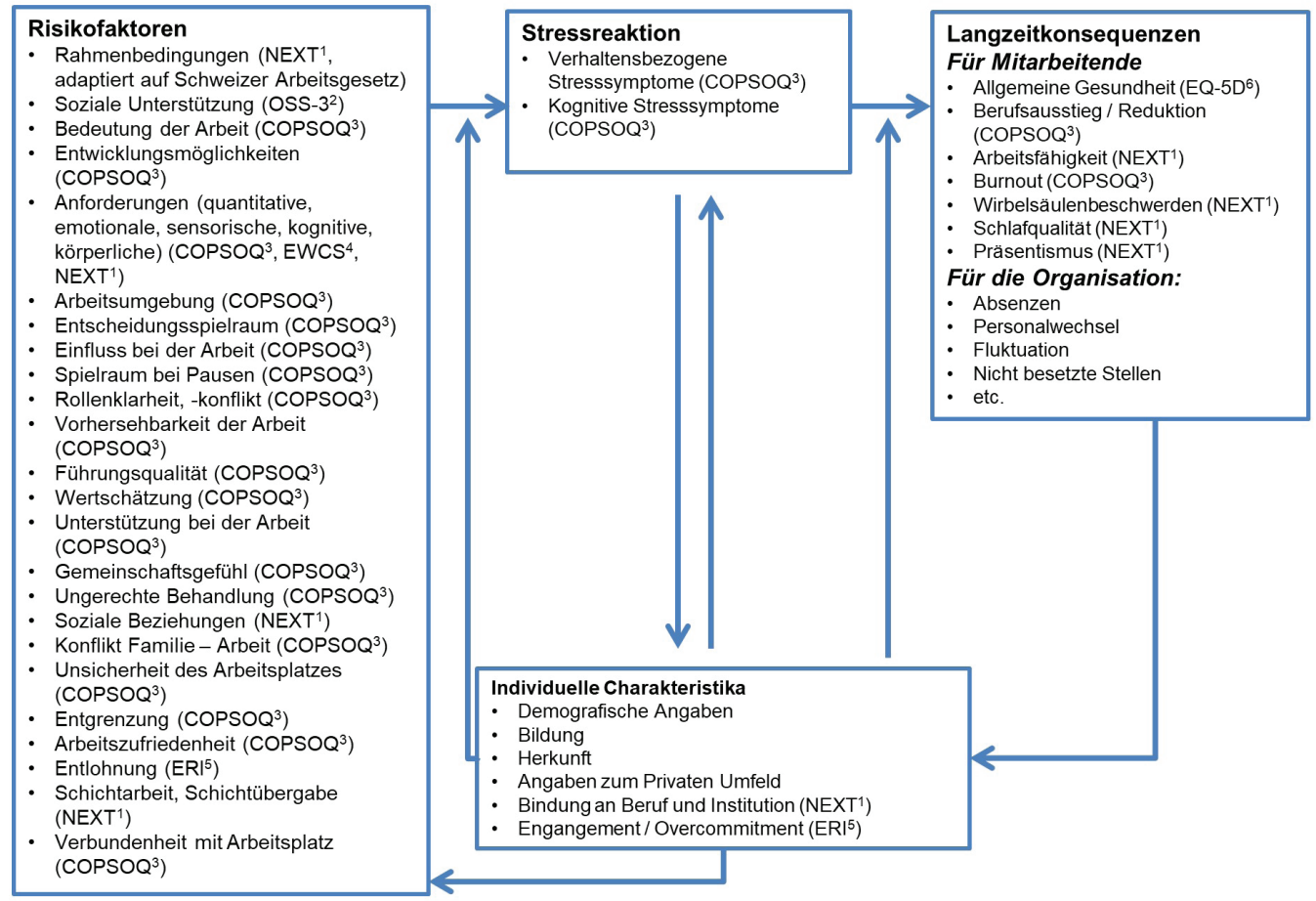

1: Nurses' early exit study; 2: The Oslo 3-Items social support scale; 3: Copenhagen Psychosocial Questionnaire; 4: European Working Conditions Surveys; 5 : Effort-Reward-Imbalance Scale; 6 : health related quality of life

Abbildung 2: Model of causes and consequences of work-related stress: STRAIN-Fragebogen (adapt. Kompier und Marcelissen (1990); Leka und Jain (2010))

\section{STRAIN-Fragebogen}

Dem STRAIN-Fragebogen liegt das „Modell of causes and consequences of work-related stress" (Kompier \& Marcelissen, 1990; Leka \& Jain, 2010) zugrunde. Basierend auf diesem Modell wurden valide und reliable Skalen ausgewählt, um potenzielle Risikofaktoren, Stressreaktionen sowie Langzeitkonsequenzen für Fachpersonen und Organisationen erfassen zu können. In Abbildung 2 werden die Themenbereiche, auf Basis des Modells, zusammengefasst dargestellt. Als Grundlage für den STRAIN-Fragebogen wurde der in der europaweiten nurses' early exit study (NEXT-Studie) verwendete Fragebogen genutzt (Hasselhorn et al., 2005). Wie der NEXT-Fragebogen, basiert auch der STRAINFragebogen somit grundsätzlich auf dem «Copenhagen Psychosocial Questionnaire» (COPSOQ) (Kristensen, 2000; Kristensen, Hannerz, Høgh \& Borg, 2005; Nübling, Stößel, Hasselhorn, Michaelis \& Hofmann, 2006) sowie zusätzlichen Skalen der «Oslo social support scale» (Oslo-3) (Sarason, Levine, Basham \& Sarason, 1983; Sarason, Sarason, Shearin \& Pierce, 1987), dem «Effort-Reward-Imbalance-Fragebogen» (ERI) (Siegrist, Li \& Montano, 2014), dem Work-Ability-Index (WAI) (Tuomi, Ilmarinen, Jahkola, Katajarinne \& Tulkki, 1998) und der Skala zu körperlicher Belastung aus dem «Sixth
European Working Conditions Survey» (EWCS Q30) (Eurofound, 2015, 2017).

Der STRAIN-Fragebogen setzt sich inhaltlich aus den folgenden verwendeten Skalen zusammen:

Insgesamt besteht derSTRAIN-Fragebogen aus 94Fragen. Davon stammen 77 Fragen aus dem NEXT-Fragebogen (Hasselhorn et al., 2005) sowie der deutschen validierten Version des COPSOQ (Nübling et al., 2006). Vier weitere Fragen des NEXT-Fragebogens wurden hinzugefügt und angepasst, da sie speziell für das Pflegepersonal entwickelt wurden und nicht generisch für das Gesundheitspersonal. Sieben Fragen wurden aufgrund der Unterschiede in Bezug auf die gesetzlichen Regelungen (Bsp. Pausen, Ruhezeiten, Pikett- und Bereitschaftsdienst) sowie auf die Ausbildungsmöglichkeiten neu erstellt. Die Skalen des COPSOQ wurden von Nübling et al. (2006) auf ihre Reliabilität (Cronbach's alpha von 0,65 bis 0,90 ) und Validität geprüft. Die psychometrischen Eigenschaften (Cronbach's alpha von 0,64 bis 0,94) der restlichen NEXT-Skalen wurden im Rahmen der Basisbefragung von Hasselhorn et al. (2005) getestet. Die Autoren der Originalskalen gaben ihre Einwilligung zur Nutzung und Anpassung.

Bei der Erstellung des STRAIN-Fragebogens wurden somit sieben Fragen neu entwickelt und vier weitere Fragen adaptiert. Diese 11 Fragen wurden kognitiv 


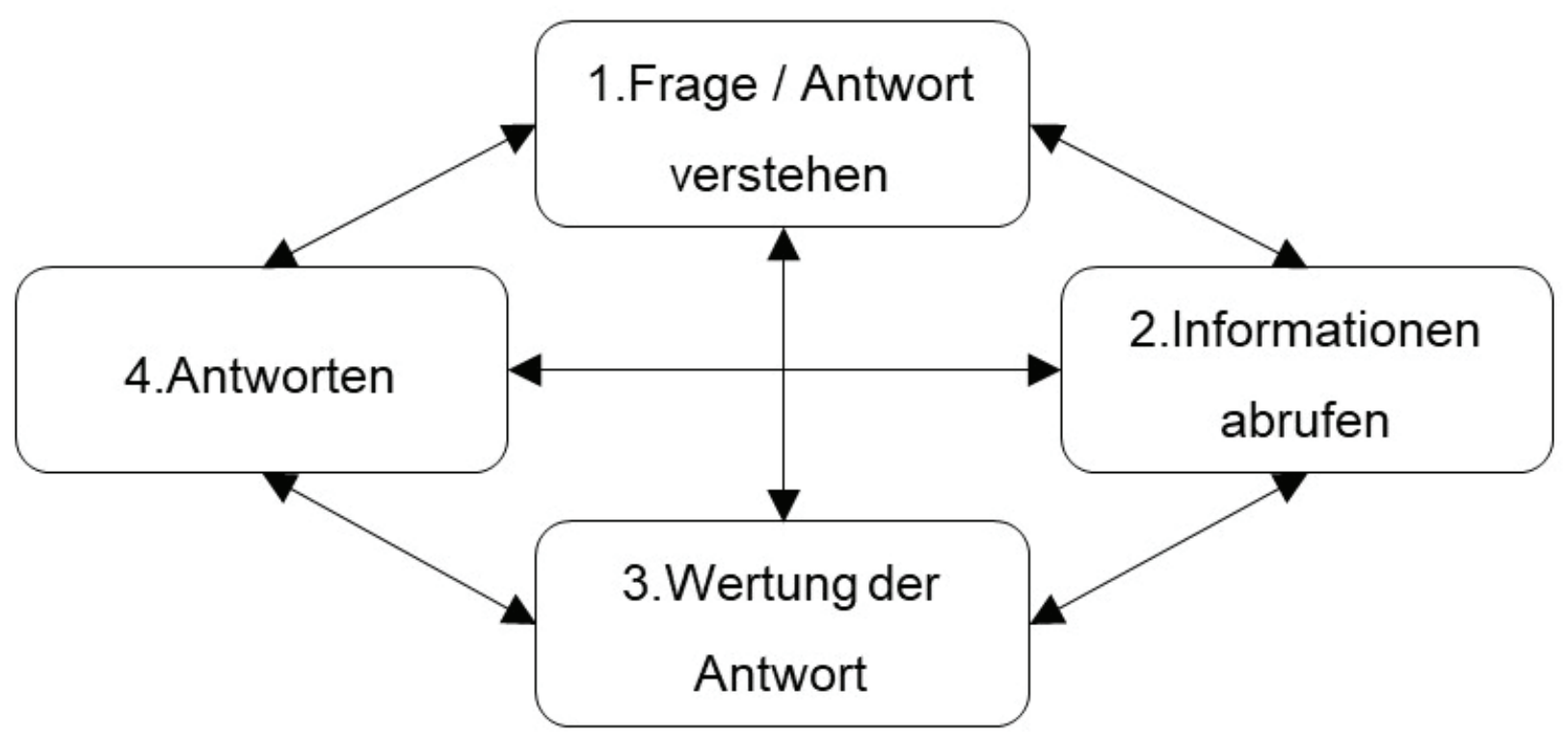

Abbildung 3: Fragen \& Antwort-Modell (Collins, 2014)

evaluiert, und abschliessend wurde der gesamte STRAINFragebogen einem Pretest unterzogen.

\section{Kognitive Interviews und Pretest}

Die Einschätzung der Verständlichkeit lässt sich aufgrund der individuellen subjektiven Wahrnehmung optimal durch Interviews erfassen (Miller, Chepp, Willson \& Padilla, 2014). Daher wurde die allgemeine Verständlichkeit mittels kognitiven Interviews nach Collins (2014) in Einzelinterviews bestimmt. Die Fragen und Antworten sollten möglichst über alle Teilnehmenden hinweg eindeutig sein, sodass eine Verallgemeinerbarkeit der Ergebnisse möglich wird (Miller et al., 2014). Prüfer und Rexroth (1996) erwähnen, dass Mängel nur in einem vorangehenden kognitiven Pretest erkannt werden können. Belson (1986) zufolge kann selbst einer plausiblen Antwort ein falsches Fragenverständnis zugrunde liegen. Antwortende können aufgrund ihrer individuellen Charakteristika (bspw. Sprachkenntnis, Fachjargon, Bildungsniveau und Erfahrung) die Fragen sowie Antworten unterschiedlich interpretieren (Miller et al., 2014). Gemäss Collins (2014) muss eine Person zur Beantwortung einer Frage vier Phasen kognitiv verarbeiten: (1) Zuerst muss die Frage mit den möglichen Antwortkategorien verstanden werden, (2) dann muss das Wissen aus der Erinnerung dazu abgerufen werden. (3) Daraufhin muss sich die Person Gedanken über die geplante Antwort machen, ob sie so formuliert werden soll bzw. ob sie die Antwort so formulieren will, um (4) die Antwort zu guter Letzt zu geben (Collins, 2014) (Abb. 3).
Nach Anpassung des Fragebogens auf Basis der Ergebnisse aus den Interviews wurde ein Pretest durchgeführt. Dieser lieferte Hinweise auf die Nutzerfreundlichkeit des Online-Fragebogens, die Verständlichkeit und logische Reihenfolge der Fragen sowie die Dauer zum Ausfüllen des Fragebogens (Prüfer \& Rexroth, 1996). Der so getestete Fragebogen wird in der nationalen STRAINStudie eingesetzt, um potenzielle Belastungsfaktoren, Stressreaktionen und Langzeitfolgen vom Schweizer Gesundheitspersonal in Akutkliniken, Psychiatrien, Rehabilitationskliniken, in der spitalexternen Pflege und in Langzeiteinrichtungen zu erheben und mittels gezielter Intervention zu reduzieren.

\section{Stichprobe und Setting}

Die Literatur verweist auf Stichprobengrössen für die kognitive Testung zwischen 10 und 25 Personen (Collins, 2014; Miller et al., 2014; Prüfer \& Rexroth, 1996; Willis, 2005). Weiter wird empfohlen, gezielt Personen mit einer hohen Bildung zu befragen, da sie „wahrscheinlicher Probleme in kurzer Zeit erkennen und artikulieren können“ (Miller et al., 2014, S. 17). Aber auch Personen mit einem tiefen Bildungsstand ermöglichen rasche Hinweise auf Probleme, wie ,schwierige Vokabeln, Fragen oder unklarer Inhalt" (Miller et al., 2014, S. 17). Da die Gesundheitsberufe verschiedene Bildungsniveaus aufweisen, musste die Stichprobe entsprechend heterogen angestrebt werden. 


\begin{tabular}{|c|c|c|c|}
\hline & Phase/Kategorie & Beispielfragen & Subkategorien nach Willis (2005) \\
\hline 1. & $\begin{array}{l}\text { Frage/Antwort } \\
\text { verstehen }\end{array}$ & $\begin{array}{l}\text { a. Was verstehen Sie unter dem Begriff } X \text { ? } \\
\text { b. In Ihren eigenen Worten, was denken Sie, versucht diese } \\
\text { Frage zu fragen? }\end{array}$ & $\begin{array}{l}\text { Der Antwortende hat Schwierigkeiten, } \\
\text { die Frage, ein bestimmtes Wort oder das } \\
\text { Konzept zu verstehen. }\end{array}$ \\
\hline 2. & Informationen abrufen & $\begin{array}{l}\text { a. Wie leicht oder schwer ist Ihnen gefallen, sich an } X z u \\
\text { erinnern? } \\
\text { b. An welche Zeitperiode haben Sie gedacht beim Beantworten } \\
\text { dieser Frage? } \\
\text { c. Wann haben Sie das letzte Mal X getan? }\end{array}$ & $\begin{array}{l}\text { Der Antwortende hat Schwierigkeiten, die } \\
\text { Informationen zur Antwort abzurufen. }\end{array}$ \\
\hline 3. & Wertung der Antwort & $\begin{array}{l}\text { a. Wie haben Sie Ihre Antwort auf diese Frage ausgearbeitet? } \\
\text { b. Wie genau ist Ihre Antwort auf Ihre Frage? } \\
\text { c. Wie haben Sie sich bei der Beantwortung dieser Frage } \\
\text { gefühlt? }\end{array}$ & $\begin{array}{c}\text { Der Antwortende hat Schwierigkeiten, die } \\
\text { Antwort zu formulieren. }\end{array}$ \\
\hline & Antworten & $\begin{array}{l}\text { a. Wie leicht oder schwer ist Ihnen die Antwort anhand } \\
\text { vorhandener Antwortmöglichkeiten gefallen? } \\
\text { b. Wieso haben Sie genau diese Antwort ausgewählt? } \\
\text { c. Vermissen Sie eine Antwortkategorie, wenn Ja, welche? }\end{array}$ & $\begin{array}{l}\text { Der Antwortende hat Schwierigkeiten, } \\
\text { die Antwort zu geben, die möglichen } \\
\text { Antworten treffen nicht zu. }\end{array}$ \\
\hline 5. & & & Nicht zuordnungsbare Probleme \\
\hline
\end{tabular}

\section{Stichprobe kognitive Interviews}

Für die kognitiven Interviews wurde eine Stichprobe von Pflegenden der spitalexternen Pflege in der deutschsprachigen Schweiz ausgewählt. Die Stichprobe wurde in drei Gruppen (Dipl. Pflegefachperson HF/ FH, Fachangestellte/r Gesundheit oder Assistent/in Gesundheit und Soziales und Pflegehelfer/in SRK) mit jeweils sechs Teilnehmenden, entsprechend des Bildungsniveaus, unterteilt. Insgesamt wurde eine Stichprobe von 18 angestrebt.

\section{Stichprobe Pretest}

Der abschliessende Pretest wurde mit insgesamt 17 Gesundheitsfachpersonen aus der Pflege $(n=12)$, den medizinisch-technischen-therapeutischen Berufen $(\mathrm{n}=3)$ sowie Personen aus dem ärztlichen Dienst $(n=2)$ durchgeführt. Die Teilnehmenden waren zum Zeitpunkt des Pretests in der ambulanten Pflege, Langzeitpflegeeinrichtungen sowie Akutspitälern und Psychiatrien tätig. Keine dieser Personen hatte zuvor an den kognitiven Interviews teilgenommen.

\section{Datensammlung kognitive Interviews}

Die Datensammlung wurde zwischen November 2016 und Januar 2017 durchgeführt. Für die kognitive Validierung des Fragebogens wurden Organisationen der spitalexternen Pflege der Deutschschweiz für die Rekrutierung von Probanden angefragt. Diese leiteten die Informationen an Interessierte weiter, welche sich beim Forschungsteam meldeten. Die Eingrenzung auf das Setting der spitalexternen Pflege erfolgte, um die Machbarkeit, mit Fokus auf die zeitlichen Ressourcen im Rahmen eines Pretests, zu gewährleisten. Auch gerade bei den neu erstellten Fragen zu den betrieblichen Strukturen (Umgang mit Pausen und Pikettdienst etc.) schien die spitalexterne Pflege für die Validierung am geeignetsten, da angenommen wurde, dass die verwendeten Begriffe für die generierten Fragen und Antwortkategorien in diesem Setting nicht geläufig seien. Für die kognitive Validierung wurde die Technik ,verbal probing“ angewandt, da die Teilnehmenden nicht, wie bei der Technik ,think-aloud“, zuerst geschult werden mussten. Bei der Technik „verbal probing" werden gegebene Antworten durch ,eine oder mehrere Zusatzfragen hinterfragt" (Prüfer \& Rexroth, 1996, S. 23). Die Zusatzfragen sind vorgängig im Interviewleitfaden notiert worden. Nach den Interviews füllten die Teilnehmenden einen kurzen Fragebogen zu ihren soziodemografischen Angaben aus. Im Interviewleitfaden wurde jeder zu prüfenden Frage pro Phase eine bis drei Fragen nach Collins (2014) zugeteilt (siehe Tabelle 1).

\section{Datensammlung Pretest}

Der Pretest des Gesamtfragebogens erfolgte online via Surveymonkey ${ }^{\circledR}$ zwischen März und Mai 2017. Es wurde eine Gelegenheitsstichprobe von Gesundheitsfachpersonen aus dem Umfeld der Forschenden durchgeführt. Sie wurden für eine Teilnahme per E-Mail oder Telefon angefragt und erhielten, bei Zusage, via E-Mail den Link zum Online-Fragebogen. Die Teilnehmenden konnten den Fragebogen ausfüllen und pro Frage ergänzend direkt kommentieren oder nach Abschluss des Fragebogens eine Rückmeldung per E-Mail an die Forschenden senden.

\section{Datenanalyse kognitive Interviews}

Die Datenanalyse erfolgte quantitativ und qualitativ. Die generierten Daten wurden mittels des 
Textverarbeitungsprogramms F4 transkribiert. Da der jeweilige Dialekt einen wesentlichen Einfluss auf die verwendeten Begriffe und somit auf das Verstehen einer Frage bzw. einer Antwort hat, wurden für das Schweizerdeutsch typische Wörter belassen.

Die qualitative Analyse geschah interpretativ nach Miller et al. (2014) in zwei Durchgängen mittels des Datenanalyseprogramms MAXQDA $12^{\circledR}$. Ziel dieses Ansatzes ist ,das konzeptuelle Verständnis über den [kognitiven] Prozess zur Formulierung einer Antwort sowie jegliche Schwierigkeit, Antwortende erfahren können [...] zu produzieren“" (Miller et al., 2014, S. 37). Insgesamt gab es fünf Analyseschritte (Interview durchführen, zusammenfassen, Vergleich der einzelnen Interviews, Vergleich zwischen den Gruppierungen, Schlussfolgerung). Mit jedem weiteren Schritt erfolgte eine Reduktion des Textumfangs sowie eine Erhöhung der Interpretationsebene.

Nach Willis (2005) gibt es fünf mögliche Subkategorien, welchen identifizierte Probleme beim Kognitiven Pretesting zugeteilt werden können (Tabelle 1, rechte Spalte). Die Zuteilung geschah im Rahmen des zweiten qualitativen Analyseschritts. Es erfolgte eine Häufigkeitsanalyse der identifizierten Probleme mittels SPSS $23^{\circledR}$ pro Subkategorie in Relation zu der Gesamtanzahl. Die maximale Anzahl möglicher Probleme entspricht der Anzahl Fragen $(n=11)$ multipliziert mit der Anzahl Teilnehmenden pro Interview-Durchgang $\left(\mathrm{n}_{1}=8 ; \mathrm{n}_{2}=10\right)$.

\section{Datenanalyse Pretest}

Die Rückmeldungen der Teilnehmenden wurden in einer Excel-Tabelle, kategorisiert nach der Frage, gesammelt. Kommentare mit gleichem Inhalt wurden zusammengefasst, sodass abschliessend pro Frage die Kommentare nach Häufigkeit sortiert werden konnten. Jede Frage wurde in der Projektgruppe besprochen und nach Konsensfindung wurden Anpassungen vorgenommen oder in der Ausgangsform belassen.

\section{ERGEBNISSE}

Insgesamt beteiligten sich 18 Pflege- und Betreuungspersonen aus der spitalexternen Pflege an der kognitiven Validierung. Die Interviews dauerten jeweils 23 bis 47 Minuten und wurden am Arbeitsplatz der Teilnehmenden oder an der Berner Fachhochschule geführt. Die grosse Mehrheit der Befragten (94,4 \%) war in einer Organisation der ambulanten Pflege tätig. Die Teilnehmenden waren im Schnitt 47 Jahre alt, mehrheitlich Schweizer Staatsbürger $(88,9 \%)$ und bis auf zwei Personen weiblich (Tabelle 2). Am abschliessenden Pretest haben 17 Personen teilgenommen. Die Teilnehmenden setzten sich aus Abteilungsleitungen,
Tabelle 2: Basismerkmale Stichprobe kognitive Interviews

\begin{tabular}{l|c|c}
\hline Charakteristika & Anzahl & $\begin{array}{c}\text { Wertebereich } \\
\text { (Mittelwert) }\end{array}$ \\
\hline Teilnehmer gesamthaft & 18 & \\
\hline Alter & & $22-61$ (47) \\
\hline Geschlecht & 16 & \\
\hline Weiblich & 2 & \\
\hline Männlich & 16 & \\
\hline $\begin{array}{l}\text { Herkunft } \\
\text { Schweiz }\end{array}$ & Je 1 & \\
\hline Arbeitsbereich & 17 & \\
\hline $\begin{array}{l}\text { ambulante Pflege } \\
\text { Freischaffende ambulante } \\
\text { Psychiatrische Pflege }\end{array}$ & 1 & \\
\hline $\begin{array}{l}\text { Bildungsabschluss } \\
\begin{array}{l}\text { Assistent/in Gesundheit und } \\
\text { Soziales }\end{array}\end{array}$ & 6 & \\
\hline $\begin{array}{l}\text { Pflegehilfe SRK } \\
\text { Fachfrau/-mann Gesundheit, }\end{array}$ & 6 & \\
\hline
\end{tabular}

Assistenz- und Oberärztinnen bzw. Assistenz- und Oberärzten, Berufsbildern/-innen, Fachfrauen/-männer Gesundheit, Heimleitungen, Physiotherapeut(inn)en, Pflegefachpersonen HF/FH, Pflegeexpert(inn)en und Pflegedienstleitenden zusammen.

\section{Ergebnisse kognitive Interviews}

Im ersten Interview-Durchgang $(\mathrm{n}=8)$ wurden insgesamt 42 Probleme in einer der vier Phasen nach Collins (2014) identifiziert, was nahezu die Hälfte (47\%) aller möglichen Antworten ausmacht. Etwas mehr als die Hälfte $(57 \%)$ der Probleme zeigte sich beim Verstehen der gesamten Frage oder eines einzelnen Begriffs und wurde der Subkategorie, „Der Antwortende hat Schwierigkeiten, die Frage, ein bestimmtes Wort oder das Konzept zu verstehen“ zugeteilt. Weitere $38 \%$ der Probleme konnten der Subkategorie „Der Antwortende hat Schwierigkeiten, die Antwort zu geben, die möglichen Antworten treffen nicht zu“ zugeordnet werden, da dort die Antwortmöglichkeiten für die spitalexterne Pflege als unzureichend oder unpassend angesehen wurden. Im zweiten Interview-Durchgang $(n=10)$ wurden zwei Probleme mit Fragen und Antworten erfasst, was $2 \%$ aller möglichen Antworten ausmacht.

Im ersten Durchgang wurden bei den Fragen 2 und 4 in keinem Interview Probleme festgestellt. Sie konnten als einheitlich verständlich eingestuft werden. Bei allen anderen Fragen ergaben sich Probleme beim Verstehen, Schwierigkeiten beim Abrufen von Informationen oder auch Schwierigkeiten mittels der vorgegebenen Antwortkategorien die Fragestellung zu beantworten. Die ursprüngliche Version der Fragen und Antworten sowie 
die Anpassungen sind in Tabelle 3 zusammenfasend dargestellt.

\section{Schwierigkeiten mit dem Verstehen}

In den Fragen $(1,3,5,6,8,11)$ zeigten sich Probleme mit dem Verständnis einzelner Wörter. Drei Fragen bzw. deren Antwortkategorien $(1,8,10)$ wurden marginal angepasst, nachdem Interviewte äusserten, einzelne Begriffe nicht $\mathrm{zu}$ verstehen oder aufgrund möglicher Mehrdeutigkeit eine falsche Antwort angekreuzt hätten (Tabelle 3). So wird bspw. ein durch den nachträglichen Titelerwerb erhaltener Bildungsabschluss nicht als solcher angesehen: „Ich habe den HF Titel geschenkt bekommen. Ich könnte [...] das HF ankreuzen. Aber ich hätte es nicht angegeben, da ich es einfach dazu bekommen habe" (I:2, 1, 16-18). Aus Frage 3 wurden zwei Fragen generiert (3, 4). Dies, da der Begriff „Beruf“" unterschiedlich interpretiert wurde: „Für mich ist aktueller Beruf „Pflege“. Ich habe die Jahre als Pflegeassistentin und als Diplomierte zusammengerechnet" (I:4, 1, 2627). Eine weitere Person äusserte sich folgendermassen dazu: „Unter Beruf verstehe ich „Berufsbezeichnung“ zum Beispiel FaGe. Weil ich vorhin Hauspflegerin geheissen habe, bin ich da jetzt unsicher, ob ich jetzt alles dazu zählen müsste" (I:6, 1, 16-19). Durch Aufteilung der Frage in „Gesundheitswesen“ und „Position“ wurde diese Mehrdeutigkeit im zweiten Durchgang erfolgreich behoben (Tabelle 3). Ausschließlich bei Frage 1 zeigte sich im zweiten Durchgang ein Verständnisproblem. So wurde der Hochschulweiterbildungsabschluss Master of Arts (MAS) als Antwortkategorie gesucht, obwohl nach den Bildungsabschlüssen und nicht nach den Weiterbildungsabschlüssen gefragt wurde: „Ich habe zum Beispiel einen MAS [...]. Diese Antwortmöglichkeit müsste da irgendwo dazwischengeschoben werden können“ (I:10, 1, 3-5). Der Antwortkategorie „Hochschulabschluss auf Masterniveau“ wurde eine Information in Klammern angefügt. So wurde versucht, der Verwechslungsgefahr von Master of Science (MSc) und Master of Arts (MAS) vorzubeugen. Frage 5 wurde im ersten Durchgang durchgängig als unverständlich oder unübersichtlich eingestuft: „Wenn die Darstellung etwas besser aufgegliedert ist, dann weiss man, um was es geht" (I:3, 1, 38-39). Von den Teilnehmenden wurde eine bessere Darstellung der Information gewünscht: „Wenn die Darstellung anders wäre, wäre es vielleicht besser" (I:2, 2, 55-56). Die Informationen zur gesetzlichen Pausenzeit wurden aus der Frage genommen und als Informationstext vorangehend integriert. Im zweiten Durchgang wurde der Informationstext als nützlich und verständlich wahrgenommen und die Frage einheitlich verstanden. Die Hälfte der Befragten im ersten Durchgang kannte den Begriff „Ruhezeit“ in Frage 6 nicht: „Pause bedeutet aufs WC rasen, eine Banane nehmen und wieder losarbeiten. Ruhezeiten [...], dass man sich an den Tisch setzt. Wir reden eigentlich gar nie von Ruhezeit" (I:3, 1, 29-30). Nach Erklärung des Begriffs wurde die Frage einheitlich verstanden. Die Frage wurde für den zweiten Durchgang so umformuliert, dass der Begriff nicht weiterverwendet werden musste. Im zweiten Durchgang lag somit eine einheitliche Verständlichkeit vor (siehe alle Anpassungen in Tabelle 3).

\section{Schwierigkeiten mit Abrufen von Informationen}

Frage 9 wurde bereits nach der ersten Phase als einheitlich verständlich eingestuft. Einzig eine Teilnehmende äusserte: „An die letzten vier Wochen erinnere ich mich nicht" (I:4, 1, 83-84). In Absprache mit der Projektgruppe wird zu Beginn des Fragebogens nun schriftlich informiert, den Arbeitsplan des letzten Monats als Unterstützung der Erinnerung beizuziehen (siehe Tabelle 3).

\section{Schwierigkeiten mit Antwortkategorien}

Bei Antworten zweier Fragen $(7,10)$ wurden nahezu in allen Interviews des ersten Durchgangs Probleme mit den Antwortkategorien festgestellt. Bei Frage 7 stellten sich die Antwortkategorien für die spitalexterne Pflege als unzutreffend heraus. Personal, welches Pikettdienst leistet, muss nicht, wie von der Antwort vorausgesetzt, bei Anruf im Betrieb, sondern beim Klienten sein: „Verständlicher wäre es aber, wenn nicht Betrieb, sondern Klienten [stehen würde]. Die Situation, dass wir in den Betrieb müssen, gibt es eigentlich nicht“ (I:2, 2, 94-95). Drei der fünf Antwortkategorien wurden somit um den Ausdruck „bei Klient(inn) en“ ergänzt. Frage 10 ist eine Vertiefungsfrage einer bereits validierten Frage zum Präsentismus des NEXTFragebogens. Die zur Frage 10 gehörige Antwort A beinhaltete zwei Antwortmöglichkeiten und wurde für den zweiten Durchgang separiert (Tabelle 3): „Das mit dem Pflichtgefühl, vielleicht schon, aber dann nicht zusammen mit, weil die Arbeit liegen bleibt. Da sind zwei Sachen zusammen" (I:6, 2, 65-66). Die dazugehörige Antwort B wurde um das Wort „Führungspersonen“ ergänzt, da ein Grund für den Präsentismus nicht nur die Rücksicht auf Kolleginnen und Kollegen, sondern auch die Führungspersonen ist: „Meine Antwort wäre um die Leitung weniger zu belasten“ (I:2, 3, 120). Im zweiten Durchgang gab es ein Problem mit einer Antwortkategorie der Frage 10. Für einen Interviewteilnehmer ,ist es nicht direkt Pflichtgefühl, sondern mehr das Verantwortungsgefühl meiner Klienten gegenüber" (I:10, 1, 36-37). Die Antwort wurde nicht angepasst (siehe alle Anpassungen Tabelle 3). 
Tabelle 3: Anpassungsprozess der Fragen \& Antwortkategorien im Verlauf der kognitiven Validierung

\begin{tabular}{|c|c|}
\hline Fragen Durchgang 1 & Angepasste Fragen/Antworten Durchgang $2^{*}$ \\
\hline $\begin{array}{ll}\text { 1. } & \text { Welches ist Ihr höchster Bildungsabschluss? } \\
\text { a. Kein Bildungsabschluss } \\
\text { b. Sekundarstufe II; Berufliche Grundbildung (Lehre)/Berufsattest/ } \\
\text { c. Fähigkeitszeugnis/ Maturität } \\
\text { d. } \quad \text { Fachhere Fachschule } \\
\text { eachschule/Universität; Hochschulabschluss auf } \\
\text { e. Fachhochschule/Universität; Hochschulabschluss auf } \\
\text { f. Masterniveau } \\
\text { g. Habtorat/PhD }\end{array}$ & $\begin{array}{l}\text { c. Tertiärstufe; Höhere Fachschule/Altrechtliche Ausbildung (DNI, } \\
\text { AKP, PsyKP)/NDS } \\
\text { d. Hochschulabschluss auf Masterniveau (konsekutiv ohne } \\
\text { f. Weiterbildung MAS) }\end{array}$ \\
\hline $\begin{array}{ll}\text { 2. } & \text { In welchem Bereich arbeiten Sie? } \\
\text { a. } & \text { Spitex } \\
\text { b. } & \text { Psychiatriespitex } \\
\text { c. } & \text { Kinderspitex } \\
\text { d. } & \text { Andere }\end{array}$ & Keine Anpassungen vorgenommen. \\
\hline $\begin{array}{l}\text { 3. Wie lange arbeiten Sie schon in Ihrem aktuellen Beruf? (Bitte } \\
\text { ohne Ausbildungszeit angeben) }\end{array}$ & $\begin{array}{l}\text { 3. Wie lange arbeiten Sie schon im Gesundheitswesen (inkl. } \\
\text { Ausbildungszeit/Praktikum)? }\end{array}$ \\
\hline
\end{tabular}

4. Wie lange arbeiten Sie schon in Ihrer aktuellen Position (Bitte ohne Ausbildungszeit angeben)

\begin{tabular}{ll}
\hline 4. & Wie lange arbeiten Sie bereits für Ihren derzeitigen Arbeitgeber? \\
\hline $5 . \quad$ Können Sie die gesetzlich festgelegten Pausen einhalten, wenn \\
Sie möchten? (1/4 Stunde bei einer täglichen Arbeitszeit von \\
mehr als $51 / 2$ Stunden; $1 / 2$ Stunde bei einer täglichen Arbeitszeit \\
von mehr als 7 Stunden; 1 Stunde bei einer täglichen Arbeitszeit \\
von mehr als 9 Stunden)
\end{tabular}
von mehr als 9 Stunden)
Keine Anpassungen.

Informationstext vor der Frage:

Im Sinne des Arbeitsgesetzes gilt jeder Arbeitsunterbruch, der von den Arbeitnehmer(inne)n zur Verpflegung und Ruhe genutzt werden kann, als Pause.

Beispiele für gesetzlich festgelegte Pausen:

- Halbtags: Bei 5.5 h Arbeitszeit pro Tag haben Sie 15 Minuten Pause zugute.

- Ganztags: Bei 7 h-9 h Arbeitszeit pro Tag haben Sie 30 Minuten

Pause zugute.

- Bei einer Arbeitszeit von mehr als 9 Stunden pro Tag haben Sie 1 Stunde Pause zugute.

6. Kommt es vor, dass Ihre tägliche Ruhezeit weniger als 9 Stunden beträgt?

7. Leisten Sie Pikett- oder Bereitschaftsdienste?

a. Nein

b. Ich leiste hauptsächlich Pikettdienst bzw. Rufbereitschaft und c. muss in mindestens 30 Minuten im Betrieb sein.

c. Ich leiste hauptsächlich Pikettdienst bzw. Rufbereitschaft und d. muss in weniger als 30 Minuten im Betrieb sein.

d. Ich leiste hauptsächlich Bereitschaftsdienste, bei welchen ich vor Ort innerhalb meines Betriebes bin.

e. Ich leiste hauptsächlich Bereitschaftsdienste, bei welchen ich zu Hause auf Abruf bin.

8. Wie häufig mussten Sie in den letzten 4 Arbeitswochen Pikettdienst leisten?

9. Hatten Sie nach Beendigung des letzten Pikettdienstes (mit oder ohne Einsatz) zwei Wochen ohne Pikettdienst?

10. Aus welchen Gründen sind Sie trotzdem zur Arbeit gegangen?

a. Pflichtgefühl und weil sonst die Arbeit liegen bleibt

b. Rücksicht auf Kolleg/in

c. Angst vor Arbeitsplatzverlust

d. Angst vor beruflichen Nachteilen

e. Andere Gründe

11. In welcher Schicht arbeiten Sie vor allem?

a. Ich arbeite gemischt in allen Schichten

b. Ich arbeite vor allem im Frühdienst

c. Ich arbeite vor allem im Spätdienst

d. Ich arbeite vor allem in der Nachtschicht

e. Ich arbeite vor allem in geteilten Diensten

*Anpassungen nach erstem Durchgang sind kursiv hervorgehoben.
8. Wie häufig mussten Sie in den letzten 4 Arbeitswochen

Information zu Beginn des Fragebogens, den Arbeitsplan des letzten Monats beizuziehen.

a.1. Pflichtgefühl

a.2. Weil sonst die Arbeit liegen bleibt

b. Rücksicht auf Kolleg(inn)en und/oder Führungspersonen

11. In welcher Schicht/welchem Dienst arbeiten Sie vor allem?

b. Ich arbeite vor allem im Frühdienst/Tagdienst

c. Ich arbeite vor allem im Spätdienst/Abenddienst Pikettdienst leisten (mit oder ohne Einsatz)? 
Im Vergleich der Gruppen nach Bildungsniveau konnte kein Unterschied in Bezug auf die Art der Probleme im Frage-Antwort-Prozess identifiziert werden. Einzig bei der Frage zur Einhaltung gesetzlicher Rahmenbedingungen hatten diplomierte Pflegefachpersonen $(n=1)$ weniger Verständnisprobleme aufgrund fehlenden Wissens als Fachfrauen/-männer Gesundheit $(\mathrm{n}=3)$.

\section{Ergebnisse Pretests}

Im Durchschnitt benötigen die Teilnehmenden 30 Minuten zum Ausfüllen des Fragebogens. Die Dauer variierte zwischen 20 und 40 Minuten. Die Teilnehmenden gaben keine Verständlichkeitsprobleme an. Sie wiesen auf Fehler der Rechtschreibung hin und gaben Optimierungsvorschläge zur Darstellung. Weiter gaben die Teilnehmenden die Rückmeldung, dass ihnen die Option zur Unterbrechung des Fragebogens und späterer Weiterführung gefehlt habe. Dies, da es während der Arbeit zu Situationen kam, die die Teilnehmenden dazu veranlassten, das Büro zu verlassen.

\section{EINSCHRÄNKUNGEN}

Die Stichprobengrösse konnte erreicht werden. Aufgrund der Gruppierung in die Bildungsniveaus ist die Gruppengrösse $(n=6)$ an der unteren Grenze des Empfohlenen (Prüfer \& Rexroth, 1996). Hinzu kommt, dass die Verteilung auf die Bereiche Spitex, Psychiatrie- und Kinderspitex nicht gleichmässig ist. Die Psychiatrie- und Kinderspitex sind untervertreten. Dies ist der Gelegenheitsstichprobe geschuldet. Eine bessere Verteilung hätte möglicherweise Hinweise auf Unterschiede zwischen den einzelnen Settings geliefert. Zudem ist zu beachten, dass die Mehrheit der Teilnehmenden $(n=16)$ Schweizer sind. Das im Ausland ausgebildete und in die Schweiz migrierte Gesundheitspersonal stammt gemäss Merçay et al. (2016) zu 16,7 \% aus Ländern mit einer anderen Sprache als einer der Schweizer Landesprachen. Dieses Personal könnte sprachliche Probleme mit dem Fragebogen haben. In dieser Studie sind sie nicht angemessen vertreten. Lediglich eine Person wies eine andere Muttersprache auf, was die gegenwertige Situation unzureichend abbildet. Jedoch muss davon ausgegangen werden, dass Pflege- und Betreuungspersonen über ein ausreichendes Sprachverständnis verfügen, um den Fragebogen ausfüllen zu können. Der Pretest wies auf potenzielle Gefahren hin. Es war den Teilnehmenden nicht möglich, die Befragung zu unterbrechen, was bei der eigentlichen Erhebung zu einer erhöhten Abbruchrate führen könnte. Zum einen wurde die durchschnittliche Dauer zum Ausfüllen des Fragebogens von 30 Minuten als lang erlebt. Zum anderen müssen Gesundheitsfachpersonen aufgrund ihrer Tätigkeit ihre Arbeit am Computer regelmässig unterbrechen.

\section{DISKUSSION}

Es konnten aus den kognitiven Interviews differenzierte Aussagen, durch Aufteilung des Frage-Antwort-Prozesses in die vier Phasen (CASM), zur Verständlichkeit der Fragen und Antwortkategorien gewonnen werden. Die Ergebnisse dieser Studie decken sich mit denen vergleichbarer Studien, die ebenfalls am häufigsten Probleme beim Verstehen aufzeigen (Willis, 2005).

Die Aussagen der Teilnehmenden lieferten Hinweise auf Mängel in den durch das Projektteam entwickelten bzw. angepassten Fragen und Antworten. Gemäss Willis (2005) sind 60-70 \% der Probleme der ersten Phase (Verstehen) zuzuordnen. Diese Unbeständigkeit bestehe, da verschiedene Forschende unterschiedliche Probleme bei der gleichen Frage identifizieren würden (Willis, 2005). Im ersten Durchgang zeigte sich, dass dieser Anteil mit $57 \%$ knapp unter den Erfahrungswerten liegt. Der Anteil der Probleme mit den Antworten ist mit $38 \%$ an zweiter Stelle, was zwar auch bisherigen Ergebnissen entspricht, jedoch nicht in diesem Ausmass (Willis, 2005). Ein Grund für den hohen Anteil der Probleme mit den Antwortkategorien könnte sein, dass die für die Entwicklung der Kategorien herangezogenen Quellen nicht für alle Gesundheitsberufe gültig waren. Als Beispiel soll die Frage 1 zum höchsten Bildungsabschluss dienen. Zur Erstellung dieser Antworten wurden die Kategorien der Schweizer Bildungsabschlüsse des BFS (2016) verwendet. Der Fakt, dass es in den letzten Jahren im Rahmen der Bildungsabschlüsse in den Pflege- und Betreuungsberufen grosse Veränderungen gab (Schäfer, Scherrer ,\& Burla, 2013), wurde darin unzureichend beachtet. Des Weiteren kann die Fülle an möglichen Aus- und Weiterbildungsabschlüssen in den Pflege- und Betreuungsberufen zu Verwirrungen führen: „Ich habe zum Beispiel einen $M A S^{\prime \prime}(\mathrm{I}: 10,1,4)$. Diese Verwechslungsgefahr von MAS und MSc musste beachtet werden, da sonst die Variable aufgrund der Mehrdeutigkeit für die Auswertung unbrauchbar wäre.

Ebenso kam es unter Verwendung von berufsspezifischen Quellen zur Erstellung von Fragen und Antwortkategorien zu Problemen während des Frage-Antwort-Prozesses. Es scheint, als seien sich die an den Interviews beteiligten Pflege- und Betreuungspersonen in der spitalexternen Pflege nicht über die gesetzlichen Regelungen zu Pause und Ruhezeit im Klaren: ,Wir haben ja eine halbe Stunde Pause. Ich könnte mir vorstellen, dass das Ruhezeit wäre“" (I:5, 1, 23-24). Auch ist die Regelung des Pikettdiensts nicht allen klar: „Zu sagen, innerhalb 30 Minuten oder mehr [beim Klienten zu sein], ist schwierig. Da bin ich jetzt gerade auch überfragt" (I:4, 69-70). Diese Erkenntnis 
deckt sich mit der Literatur, dass viele Pflegende die für sie relevanten gesetzlichen Rahmenbedingungen nicht kennen (Wagner, 2010). Wagner (2010) zufolge habe dies mit dem geringen Stellenwert des Themas in der Ausbildung bzw. im Studium zu tun. Direkte Hinweise dazu konnten die Interviews nicht liefern. Der Vergleich nach Bildungsabschluss zeigte jedoch, dass die Fachfrauen Gesundheit weniger über die gesetzlichen Regelungen zu Pause und Ruhezeiten wussten als Dipl.Pflegefachpersonen.

Anscheinend kann auch nicht impliziert werden, dass für einen geläufigen Begriff Übereinstimmung in der Deutung vorliegt (Boggatz, 2015). Gemäss Bonis (2013) ist die Übereinkunft über einen Begriff kulturell bedingt, die sich auch über die Zeit verändern kann. Aus sprachwissenschaftlicher Sicht gibt es zum Beispiel einen klaren Unterschied zwischen Verantwortungsgefühl und Pflichtgefühl. Beide Begriffe werden jedoch im Alltag, seit Längerem, meist als synonym verstanden (Fromm, 2015). Ein weiteres Beispiel ist der Begriff „Beruf“, der „bis heute vielschichtig, mehrdeutig und umstritten ist" (Dostal, Stooß \& Troll, 1998, S. 440). Ein Grund hierfür könnte die Dissolution des Begriffs durch Überschneidungsbereiche der Berufsfelder in den Pflege- und Betreuungsberufen als auch anderen Gesundheitsberufen sein. Diese Überschneidungen bestehen gemäss Bollinger, Gerlach und Pfadenhauer (2016) aufgrund der Akademisierung nicht-ärztlicher Gesundheitsberufe sowie der Anpassungen an gesellschaftliche Bedarfe. Die Aufteilung der Frage 3 ,Wie lange arbeiten Sie schon in Ihrem aktuellen Beruf?" in zwei neue Fragen ermöglichte das Separieren der zuerst identifizierten Mehrdeutigkeit. Aufgrund der Rückmeldungen aus dem Pretest wurden weitere Anbieter für Online-Fragebögen gesichtet, die in ihren Programmen die Option zur Unterbrechung der Teilnahme ermöglichen. Diese setzten jedoch technisch voraus, dass nicht gleichzeitig an einem Endgerät zwei Fragebögen ausgefüllt werden können. Da in einigen Gesundheitsorganisationen jedoch das Vorhandensein eines einzigen Computers pro Station noch geläufig ist, sollte bei der eigentlichen Erhebung das Programm entsprechend den Bedürfnissen und Möglichkeiten der teilnehmenden Organisation gewählt werden.

\section{SCHLUSSFOLGERUNG}

Die kognitiven Interviews zeigen klar auf, dass eine einheitliche Verständlichkeit von Items in Fragebögen nicht selbstverständlich ist. Gerade bei einer heterogenen Zielgruppe, wie dem Gesundheitspersonal, ist eine unterschiedliche Verständlichkeit in Bezug auf die Fragebogenitems anzunehmen. Ein einheitlich verständlicher Fragebogen ist jedoch für berufsübergreifende Vergleiche sowie interprofessionell zu planende Massnahmen essenziell. Durch kognitive Interviews und Pretest kann dies erreicht werden. Um die Gültigkeit der Resultate auf Basis von Fragebögen zu erhöhen, scheinen kognitive Pretests angebracht. Durch die Testung in dieser Studie konnte eine einheitliche Verständlichkeit des STRAIN-Fragebogens zur Erfassung der Arbeitsbelastung bei den Gesundheitsfachpersonen erreicht werden.

Erstmalig liegt ein umfassender Fragebogen zur Erfassung der Arbeitsbelastung und dessen Folgen beim Gesundheitspersonal für die Schweiz vor. Die einheitliche Verständlichkeit ist auch hier im Kontext der Zeit und somit der sich verändernden Kultur zu verstehen. Beispielsweise werden sich die Professionalisierung und Akademisierung diverser Gesundheitsberufe auf die Verständlichkeit auswirken. Die Halbwertszeit dieser Gültigkeit lässt sich prospektiv nicht bestimmen. So scheint es angebracht, den STRAIN-Fragebogen nach geraumer Zeit einem erneuten kognitiven Pretest zu unterziehen.

\section{DANK}

Der Autor und die Autorinnen danken den SpitexOrganisationen für ihre Unterstützung in der Rekrutierung. Ein besonderes Dankeschön geht an die Gesundheitsfachpersonen, die bereit waren, an den Interviews sowie dem Pretest teilzunehmen und somit einen wesentlichen Beitrag an den Vorarbeiten zum Projekt STRAIN geleistet haben.

\section{ETHISCHE PRÜFUNG}

Im Rahmen der STRAIN-Studie wurde die Ethikkommission des Kantons Bern um Bewilligung der Studie angefragt. Sie erklärte sich nicht zuständig für die Studie, da diese nicht unter das Humanforschungsgesetz fällt. Die Nicht-Zuständigkeitserklärung wurde am 24.10.2016 ausgestellt. Die Teilnehmenden sind Fachpersonen, die eigenverantwortlich über die Teilnahme entscheiden können. Die Beteiligten wurden schriftlich und mündlich über Inhalt/Zielsetzung sowie zur Freiwilligkeit der Teilnahme aufgeklärt. Ihr Einverständnis wurde schriftlich eingeholt. Der Datenschutz wurde durch Pseudonymisierung der Daten gewährleistet.

\section{INTERESSENKONFLIKTE}

Es liegt kein Interessenkonflikt vor. Die Arbeiten erfolgten im Rahmen des nationalen 
Projekts „Strategie gegen den Fachkräftemangel in den Gesundheitsberufen", gefördert durch das Staatssekretariat für Bildung, Forschung und Innovation und die fünf beteiligten öffentlichen Fachhochschulen.

Literaturverzeichnis

Aiken, L. H., Sermeus, W., Van den Heede, K., Sloane, D. M. Busse, R., McKee, M., Bruyneel, L., Rafferty, A. M., Griffiths, P., Moreno-Casbas, M. T., Tishelman, C., Scott, A., Brzostek, T., Kinnunen, J., Schwendimann, R., Heinen, M., Zikos, D., Sjetne, I. S., Smith, H. L. \& Kutney-Lee, A. (2012). Patient safety, satisfaction, and quality of hospital care: cross sectional surveys of nurses and patients in 12 countries in Europe and the United States. British Medical Journal, 344, e1717. doi: 10.1136/bmj.e1717.

Belson, W. A. (1986). Validity in Survey Research. Aldershot: Gower.

Berner Fachhochschule. (2017). STRAIN - Work-related Stress among health professionals in Switzerland. Abgefragt am 18.06.2018, unter http://gesundheit.bfh.ch/strain.

Boggatz, T. (2015). Begriffsanalysen in der Pflegewissenschaft. Journal für Qualitative Forschung in Pflege- und Gesundheitswissenschaft, 2(2).

Bolliger, C., Golder, L., Jans, C. \& Rüefli, C. (2016). Der Ausstieg aus der kurativen ärztlichen Tätigkeit - Schlussbericht. Bern: gfs.bern.

Bollinger, H., Gerlach, A. \& Pfadenhauer, M. (2016). Gesundheitsberufe im Wandel. Soziologische Betrachtungen und Interpretationen (Vol. 4). Frankfurt: Mabuse.

Bonis, S. A. (2013). Concept analysis: method to enhance interdisciplinary conceptual understanding. Advances in Nursing Science, 36(2), 80-93. doi: 10.1097/ ANS.0b013e318290d86e.

Bundesamt für Statistik. (2016). Bildungsabschlüsse Ausgabe 2016. Neuchâtel: Bundesamt für Statistik, unter https://www. bfs.admin.ch.

Collins, D. (2014). Cognitive interviewing practice: Sage.

Dostal, W., Stooß, F. \& Troll, L. (1998). Beruf Auflösungstendenzen und erneute Konsolidierung. Mitteilungen aus der Arbeitsmarkt- und Berufsforschung, 31(3), 438-460.

Eurofound. (2015). Sixth European Working Conditions Survey. Luxembourg: Publications Office of the European Union.

Eurofound. (2017). European Working Conditions Survey, 2015 [data collection]. 4th edition. UK Data Service. SN: 8098, unter http://doi.org/10.5255/UKDA-SN-8098-4.

Fromm, E. (2015). Die Revolution der Hoffnung - Für eine Humanisierung der Technik (R. Funk Ed.): Open Publishing.

Glazer, S. \& Beehr, T. A. (2005). Consistency of implications of three role stressors across four countries. Journal of Organizational Behavior, 26(5), 467-487.

Hahn, S., Richter, D., Beck, M. \& Thilo, F. (2013). Panorama Gesundheitsberufe 2030. Bern: Berner Fachhochschule, unter http://www.bag.admin.ch/.
Hasselhorn, H. M., Müller, B. H., Tackenberg, P., Kümmerling, A. \& Simon, M. (2005). Berufsausstieg bei Pflegepersonal: Arbeitsbedingungen und beabsichtigter Berufsausstieg bei Pflegepersonal in Deutschland und Europa. Bremerhaven: Wirtschaftsverlag NW, Verlag für Neue Wissenschaften.

Kompier, M. A. J., \& Marcelissen, F. H. G. (1990). Handbook of Work Stress: A Systematic Approach for Organizational Practice. Amsterdam: NIA.

Kristensen, T. S. (2000). A new tool for assessing psychosocial factors at work: The Copenhagen Psychosocial Questionnaire. Copenhagen: National Institute of Health, unter www.arbejdsmiljoforskning.dk.

Kristensen, T. S., Hannerz, H., Høgh, A. \& Borg, V. (2005). The Copenhagen Psychosocial Questionnaire-a tool for the assessment and improvement ofthe psychosocial work environment. Scandinavian Journal of Work, Environment \& Health, 31(6), 438-449.

Leka, S., \& Jain, A. (2010). Health impact of psychosocial hazards at work: An overview.

Martikainen, P., Bartley, M. \& Lahelma, E. (2002). Psychosocial determinants of health in social epidemiology. International Journal of Epidemiology, 31(6), 1091-1093. doi: 10.1093/ ije/31.6.1091.

Merçay, C., Burla, L. \& Widmer, M. (2016). Gesundheitspersonal in der Schweiz. Bestandesaufnahme und Prognosen bis 2030 (Obsan Bericht 71). Neuchâtel: Schweizerisches Gesundheitsobservatorium, unter http://www.obsan.admin. $\mathrm{ch} /$.

Michie, S. \& Williams, S. (2003). Reducing work related psychological ill health and sickness absence: a systematic literature review. Occupational and Environmental Medicine, 60(1), 3-9. doi: 10.1136/oem.60.1.3.

Miller, K., Chepp, V., Willson, S. \& Padilla, J. L. (2014). Cognitive Interviewing Methodology. New York: John Wiley.

Nübling, M., Stößel, U., Hasselhorn, H.-M., Michaelis, M. \& Hofmann, F. (2006). Methoden zur Erfassung psychischer Belastungen: Erprobung des COPSOQ in Deutschland. Psycho-Social-Medicine, 3, 1-14.

Prüfer, P. \& Rexroth, M. (1996). ZUMA-Arbeitsbericht Nr.96/05. Verfahren zur Evaluation von Survey - Fragen: Ein Überblick. Mannheim, unter http://www.gesis.org/.

Rüesch, P., Bänziger, A., Dutoit, L., Gardiol, L., Juvalta, S., Volken, T., \& Künzi, K. (2014). Prognose Gesundheitsberufe Ergotherapie, Hebammen und Physiotherapie 2025. ZHAW Reihe Gesundheit Nr. 3. Wintherthur: ZHAW, unter https:// www.zhaw.ch.

Sarason, I. G., Levine, H. M., Basham, B. R. \& Sarason, B. R. (1983). Assessing Social Support: The Social Support 
Questionnaire. Journal of Personality and Social Psychology, 44(1), 127-139.

Sarason, I. G., Sarason, B. R., Shearin, E. N. \& Pierce, G. R. (1987). A brief measure of social support: Practical and theoretical implications. Journal of Social and Personal Relationships, 4, 497-510.

Schäfer, M., Scherrer, A. \& Burla, L. (2013). Bildungsabschlüsse im Bereich Pflege und Betreuung. Systematische Übersichtsarbeit (Obsan Dossier 24). Neuchâtel: Schweizerisches Gesundheitsobservatorium, unter http:// www.obsan.admin.ch/.

Schubert, M., Schaffert-Witvliet, B., De Geest, S., Glass, T., Aiken, L., Sloane, D. M., Clarke, S. \& Abraham, I. (2005). RICH - Nursing Study. Rationing of Nursing Care in Switzerland $=\mathrm{CH}$. Basel: Institut für Pflegewissenschaft im Auftrag des Bundesamts für Gesundheit, unter http://www. bag.admin.ch/.

Siegrist, J., Li, J. \& Montano, D. (2014). Psychometric properties of the effort-reward imbalance questionnaire. Department of Medical Sociology, Faculty of Medicine, Düsseldorf University, Germany.

Suadicani, P., Olesen, K., Bonde, J. P. \& Gyntelberg, F. (2014). Psychosocial work conditions associated with sickness absence among hospital employees. Occupational Medicine, 64(7), 503-508. doi: 10.1093/occmed/kqu064.

Tuomi, K., IImarinen, J., Jahkola, A., Katajarinne, L. \& Tulkki, A. (1998). Work Ability (2. Aufl.). Helsinki: Finnish Institute of Occupational Health.

Wagner, P. A. (2010). Paranoia ist ebenso wenig gerechtfertigt wie Sorglosigkeit. Curaviva, 4(10), 40-43.

Wenderlein, F. U. \& Schochat, T. (2003). Betriebsbedingte Belastungen bei Pflegekräften-Auswirkungen auf Arbeitszufriedenheit und Fehlzeiten. Eine empirische Studie an 861 Probanden. Zeitschrift für Arbeitsmedizin, Sozialmedizin und Umweltmedizin, 38, 262-269.

Willis, G. B. (2005). Cognitive Interviewing: A Tool for Improving Questionnaire Design. Thousand Oaks (CA): Sage Publications. 